\title{
Rate-Distortion Optimized Hybrid Error Control for Real-Time Packetized Video Transmission
}

\author{
Fan Zhai, Yiftach Eisenberg, Thrasyvoulos N. Pappas, Randall Berry, and Aggelos K. Katsaggelos \\ Department of Electrical and Computer Engineering, Northwestern University \\ 2145 Sheridan Road, Evanston, IL 60208, USA \\ Email: \{fzhai, yeisenbe, pappas, rberry, aggk\}@ece.northwestern.edu
}

\begin{abstract}
We study hybrid error control for real-time video transmission. The study is carried out using a proposed integrated joint source-channel coding framework, which jointly considers error resilient source coding, channel coding, and error concealment, in order to achieve the best video quality. We focus on the performance comparison of several error correction scenarios, such as forward error correction (FEC), retransmission, and the combination of both. Simulation results show that either FEC or retransmission can be optimal depending on the packet loss rates and network round trip time. The proposed hybrid FEC/retransmission scheme outperforms both.
\end{abstract}

\section{INTRODUCTION}

Real-time video applications, such as on-demand video streaming, videophone and videoconferencing, have gained increased popularity. However, it is well known that the besteffort design of the current Internet makes it difficult to provide the quality of service (QoS) needed by these applications. Error control implemented in different network layers is fundamental in the design of a multimedia communication system. In this work, we study application-layer error control. Specifically, at the sender side, we consider error resilient source coding, hybrid forward error correction and applicationlayer retransmission, and at the receiver side, we consider error concealment. We present an integrated joint source channel coding (IJSCC) framework that jointly considers these error control components to achieve the best video quality.

Each of the above error control approaches is designed to deal with a lossy packet channel. Error resilient source coding accomplishes this by adding redundancy at the source coding level to prevent error propagation and limit the distortion caused by packet losses. In this paper, we consider optimal mode selection (prediction mode and quantizer) to achieve this [1], [2]. Another way to deal with packet loss is to use error correction techniques in the application/transport layer. Two basic techniques are used: Forward Error Correction (FEC) and Automatic Repeat reQuest (ARQ). Each has its own benefits in error robustness and network traffic load [3], [4]; we will consider both approaches in the IJSCC framework. Finally, error concealment refers to post-processing techniques employed by the decoder to recover from packet loss by utilizing the spatial and temporal correlation of the video sequence.

For error correction, FEC is usually preferred for real-time video applications due to the strict delay requirements and semi-reliable nature of video streams [5], [6]. Joint source coding and FEC has been extensively studied in the literature [5]-[8]. However, if an application has a relatively loose end-to-end delay constraint (e.g., on-demand video streaming applications) or the round-trip-time (RTT) is short with respect to the maximum allowable delay (as in a LAN), retransmission can be more applicable. This is because ARQ can adapt automatically to the channel loss characteristics by retransmitting only lost packets.

We focus on the application layer error control. Specifically, we study different error control scenarios including pure FEC, pure ARQ, and hybrid FEC/retransmission. Our goal is to find an optimal error control scheme for video transmission in different network situations (such as packet loss probability and network round trip time) and application requirements (such as end-to-end delay).

In related work, in [9], a general cost-distortion framework has been proposed to study several scenarios such as DiffServ, sender-driven retransmission and receiver-driven retransmission. In the IJSCC framework, we take into account source coding and error concealment, which are not considered in [9]. In terms of hybrid FEC/retransmission, for wireless IP networks, a link-layer hybrid FEC/ARQ scheme is considered in [10] and an application-layer hybrid FEC/ARQ technique is proposed for video transmission in [3], which is based on heuristic methods. On the other hand, a receiver-driven hybrid FEC/Pseudo-ARQ mechanism is proposed for Internet multimedia multicast in [4]. In [11], pure ARQ is used for the base layer and pure FEC is used to protect the enhancement layer for wireless scalable video transmission. Podolsky et al. [12] also consider optimal delay-constraint ARQ for streaming preencoded layered video. Our work differs from the above in that we jointly consider FEC and application-layer sender-driven retransmission, where lost packets are selectively retransmitted to achieve rate-distortion optimization.

\section{PRELIMINARIES}

\section{A. Real-Time Video Transmission System}

In a real-time video transmission system, video packets (referred to as source packets) are first generated by a video encoder. In the application layer, parity check packets used for FEC may be generated. In addition, lost packets may be retransmitted if applicable. After passing through the network protocol stack (e.g. RTP/UDP/IP), transport packets are formed to be sent over a packet-switched network. We 
define an initial setup time, $T_{\max }$, as the duration between the moment the first packet is transmitted at the encoder and its playback at the decoder. The longer the initial setup time, the more robust the video packets are to the channel variations, but the larger the decoder buffer required. For the real-time application, packets arriving at the receiver later than the scheduled playback time are discarded. Lost packets may be concealed at the decoder.

In our simulations, packet loss is modeled by a Bernoulli process, i.e., each packet is independently lost with probability $\epsilon$. We assume the network delay is constant for simplicity. We further assume that the receiver responds to a lost/corrupted packet with a negative acknowledgement, and responds to a correctly received packet with a positive acknowledgement. All acknowledgements are assumed to arrive correctly.

\section{B. Joint source-channel coding}

Different source coding parameters and error protection ratios result in different levels of coding efficiency and robustness. Joint source-channel coding (JSCC) aims at finding the optimal trade-off between these factors. Let $\boldsymbol{\mu}=$ $\left\{\mu_{1}, \mu_{2}, \ldots, \mu_{M}\right\} \in \mathcal{Q}$ and $\boldsymbol{\nu}=\left\{\nu_{1}, \nu_{2}, \ldots, \nu_{M}\right\} \in \mathcal{R}$ denote the vector of source coding parameters and channel coding parameters for the $M$ source packets in a frame, respectively. The objective of JSCC is to minimize the total expected distortion for the $n$-th frame given the transmission delay constraint, i.e.,

$$
\min _{\{\boldsymbol{\mu} \in \mathcal{Q}, \boldsymbol{\nu} \in \mathcal{R}\}} E\left[D^{(n)}(\boldsymbol{\mu}, \boldsymbol{\nu})\right] \quad \text { s.t. } \quad B^{(n)} / R_{T} \leq T_{0}^{(n)},
$$

where $B^{(n)}$ is the total bits used for both source and channel coding, $R_{T}$ is the transmission rate, and $T_{0}^{(n)}$ is the transmission delay constraint for this frame. Since video packets are usually of different importance, the solution to (1) will be a bit allocation that varies across video packets, leading to different packets receiving unequal error protection (UEP).

\section{Expected Distortion}

The expected distortion of the $k$-th source packet is

$$
E\left[D_{k}\right]=\left(1-\rho_{k}\right) E\left[D_{R, k}\right]+\rho_{k} E\left[D_{L, k}\right],
$$

where $E\left[D_{R, k}\right]$ and $E\left[D_{L, k}\right]$ are the expected distortion when this packet is either received correctly or lost, respectively, and $\rho_{k}$ is its loss probability. The relationship between the source packet loss probability and transport packet loss probability depends on the specific packetization scheme chosen. Note that both $D_{L, k}$ and $D_{R, k}$ are random variables. This is because, due to channel losses, the reference frames at the decoder and the encoder may not be the same.

The distortion measurement is based on a per-pixel distortion calculation, which ensures accurate estimation of the overall end-to-end distortion [1], [2]. Assuming the mean squared error (MSE) criterion, an algorithm called ROPE (Recursive Optimal Per-pixel Estimate) [2] is used to recursively calculate the overall expected distortion level of each pixel. The image quality measure used is the peak signal to noise ratio (PSNR), defined as $\mathrm{PSNR}=10 \log \frac{255^{2}}{\mathrm{MSE}} \mathrm{dB}$.

\section{Packetization}

In [8], different packetization schemes are studied for FEC in Internet video transmission. Here, we employ packetization scheme 1 in [8]. In this packetization, one GOB (group of blocks) ${ }^{1}$ is packetized into one source packet, which is directly packetized into one transport packet by the attachment of a transport packet header. Thus, each GOB is independently decodable. Parity packets are generated in addition to source packets to perform inter-packet FEC. The same packetization scheme is used in [3], [4], [7], [11].

\section{E. Hybrid FEC and Selective Retransmission}

In this work, we consider systematic Reed-Solomon (RS) codes to recover packet losses, but the basic framework could easily be applied to other erasure codes as well. We group $M$ source packets in frame $n$ into one block and protect the block with $\mathrm{RS}(N, M)$ code, where $(N-M)$ is the number of parity packets. Note that $N$ may vary from frame to frame. A source packet is regarded as lost after error recovery at the receiver only when the corresponding transport packet is lost and the block containing the lost transport packet cannot be recovered. Thus, the probability of source packet loss $\rho$ after error recovery is defined as $\rho=\sum_{i=N-M+1}^{N} \frac{i}{N}\left(\begin{array}{c}N \\ i\end{array}\right) \epsilon^{i}(1-\epsilon)^{N-i}$, where $\epsilon$ is the probability of transport packet loss.

Even with UEP, FEC cannot achieve the capacity of the packet erasure channel and completely avoid packet loss, due to the limit on the block size from the delay constraints. FEC incurs constant transmission overhead even when the channel is loss free. In addition, the appropriate protection of FEC depends on the accuracy of channel state estimate. On the other hand, ARQ can automatically adapt to the varying channel by transmitting only as many redundant packets as lost packets. For near real-time applications, delay constrained application-layer ARQ has been considered and proved to be useful for video streaming in some situations [3], [9], [11], [12]. In this work, we consider the hybrid of FEC and selective retransmission to perform optimal error control.

\section{INTEGRATED JOINT SOURCE-CHANNEL CODING}

\section{A. Problem Formulation}

Assume that the encoder buffer can accommodate $A+1$ frames, where $A$ is the number of frames that are eligible to be retransmitted. Let $\sigma_{k}^{(n)} \in\{0,1\}$ denote the retransmission parameter for the $k$-th source packet in frame $n$, where 0 denotes no retransmission and 1 denotes retransmission. Let $\boldsymbol{\sigma}^{(\boldsymbol{n})}=\left\{\sigma_{1}^{(n)}, \ldots, \sigma_{M}^{(n)}\right\}$ denote the retransmission parameter vector for frame $n$, and $\boldsymbol{\sigma}=\left\{\boldsymbol{\sigma}^{(n-A)}, \ldots, \boldsymbol{\sigma}^{(n-1)}\right\}$ the vector for the $A$ frames. Real-time video applications usually impose strict delay constraints on when each frame is displayed at the receiver. This is achieved through higher-level rate control that typically assigns a bit budget per frame. For simplicity, we assume the transmission delay for the $n$-th frame, $T_{0}^{(n)}$, is given and therefore known. Thus, the objective of the IJSCC

${ }^{1}$ Following the H.263 standard, we use a GOB to denote one row of MBs (macro-blocks). 
is to minimize total expected distortion of the $A+1$ frames in the encoder buffer subject to the delay constraint by optimally allocating bits to source coding, FEC, and retransmission,

$$
\begin{aligned}
\min _{\{\boldsymbol{\mu}, \boldsymbol{\nu}, \boldsymbol{\sigma}\}} & \sum_{i=1}^{A} E\left[D^{(n-i)}\left(\boldsymbol{\sigma}^{(\boldsymbol{n}-\boldsymbol{i})}\right)\right]+\sum_{k=1}^{M} E\left[D_{k}^{(n)}(\boldsymbol{\mu}, \boldsymbol{\nu})\right] \\
\text { s.t. } & \sum_{i=1}^{A} \sum_{k=1}^{M} \sigma_{k}^{(n-i)} T_{k}^{(n-i)}+\sum_{k=1}^{M} T_{k}^{(n)} \leq T_{0}^{(n)} .
\end{aligned}
$$

The FEC parameter set is defined as $\mathcal{R}=\left\{\left(N_{1}, M\right), \ldots,\left(N_{q}\right.\right.$, $M)\}$, where $q$ is number of available code options. Gains might be obtained by grouping the retransmitted packets and the packets in the current frame together to perform FEC. However, this introduces additional delay for the retransmitted packets. Thus, we only consider FEC for the current frame.

The above formulation is for an optimization scheme with a sliding window of size $A+1$ frames. The optimization window shifts at the frame level instead of at the packet level, since the latter usually leads to much higher computational complexity. In addition, the packets in one frame typically have the same deadline for playback. In this formulation, upon the processing of each frame, the optimization (retransmission policy for the first $A$ frames based on feedback, and source coding and FEC for the current frame) is performed on the $A+1$ frames in the window. After optimization is done, the window shifts forward by one frame, and the optimization is reinitiated based on the updated feedback.

Based on the received feedback, the probability of packet loss for all the past $A$ frames are updated accordingly. For example, if one packet is known to be received, its probability of loss becomes 0 ; if one is lost, its loss probability becomes 1 if no further retransmission for this packet has been acknowledged. Based on the updated probability of packet loss information, the expected distortion of all packets in the encoder buffer is recursively re-calculated as in [2]. In using this model, the error propagation due to packet loss can be fully captured and consequently the effect of previously lost packets on the future frames is taken into account. Since each time we do not consider re-encoding the past $A$ frames, the complexity in updating the expected distortion is negligible. Additional gain may be obtained by considering the future frames when the current frame is encoded. However, this leads to a very complicated and usually intractable problem. In addition, for a real-time application, future frames are not always available when the current frame is encoded.

Next, we discuss how to calculate the probability of packet loss $\rho_{k}$ in order to find the expected distortion in (2). For a packet in the current frame, the probability of packet loss can be defined as $\rho_{k}^{(n)}=\rho_{k, F E C}^{(n)} \rho_{k, R E T}^{(n)}$, where $\rho_{k, F E C}^{(n)}$ and $\rho_{k, R E T}^{(n)}$ denote the probability of packet loss due to FEC and retransmission, respectively. $\rho_{k, F E C}^{(n)}$ is defined in Sec. II-E, and $\rho_{k, R E T}^{(n)}=\epsilon^{m}$, where $m$ denotes the average retransmission times. Because lost packets are selectively retransmitted, $m$ is not a constant and is not known when the current frame is encoded. In addition, $m$ is dependent on how $\rho_{k, R E T}^{(n)}$ itself is calculated and the video content as well. In this work, we use an estimate $\tilde{m}$ in the optimization. Figure 1 shows the performance of the hybrid FEC/retransmission system versus $m$ for the Foreman test sequence. Based on these results, we use $\tilde{m}=\frac{A}{(1+\mathrm{RTT})^{2}}$, where RTT is in the unit of one frame's duration $T_{F}$; this appears to provide good results and is used subsequently. Note that the maximum number of available retransmission opportunities is $\lfloor A /(1+\mathrm{RTT})\rfloor$. In addition, from Fig. 1, we can see that the system performance is not very sensitive to the choice of $\tilde{m}$.

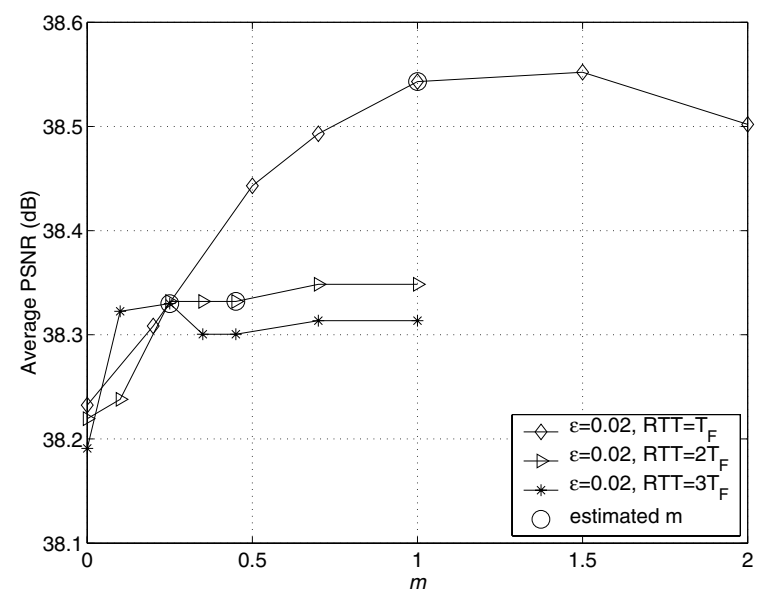

Fig. 1. Average PSNR vs. $m$ in the hybrid FEC/retransmission system. (QCIF Foreman sequence at $F=15 \mathrm{fps}, R_{T}=480 \mathrm{kbps}$ and $A=4$ )

In considering possible retransmission of the packets in the current frame, the expected additional transmission delay used for retransmission in the future should be taken into account, which is calculated by $E\left[\Delta T_{k}^{(n)}\right]=\sum_{k=1}^{M} \tilde{m} \rho_{k, F E C}^{(n)} T_{k}^{(n)}$. The delay constraint in (3) can be modified correspondingly.

For a lost packet in the past frames, we let $\rho_{k}^{(n-i)}=$ $\rho_{k, U P D}^{(n-i)} \rho_{k, R E T}^{(n-i)}$ for $i=1, \ldots, A$, where $\rho_{k, U P D}^{(n-i)}$ is the updated probability of packet loss based on feedback and $\rho_{k, R E T}^{(n-i)}$ is the probability of packet loss due to retransmissions. Assume that one past frame is protected by an $\operatorname{RS}(N, M)$, and $L$ packets are lost. Let $J=L+M-N$ and $V$ be the number of retransmitted packets in that frame. The calculation of $\rho_{k, R E T}^{(n-i)}$ is different for the lost packets that are either retransmitted or those that are not. If $V<J$, we have

$$
\rho_{k, R E T}^{(n-i)}=\epsilon^{\sigma_{k}^{(n-i)}}
$$

if $V=J$, we have

$$
\rho_{k, R E T}^{(n-i)}= \begin{cases}\epsilon & \text { if } \sigma_{k}^{(n-i)}=1 \\ 1-(1-\epsilon)^{J} & \text { if } \sigma_{k}^{(n-i)}=0\end{cases}
$$

and if $V>J$ we have

$\rho_{k, R E T}^{(n-i)}= \begin{cases}\sum_{j=V-J+1}^{V} \frac{j}{V}\left(\begin{array}{c}V \\ j\end{array}\right) \epsilon^{j}(1-\epsilon)^{V-j} & \text { if } \sigma_{k}^{(n-i)}=1 \\ \sum_{j=V-J+1}^{V}\left(\begin{array}{c}V \\ j\end{array}\right) \epsilon^{j}(1-\epsilon)^{V-j} & \text { if } \sigma_{k}^{(n-i)}=0 .\end{cases}$ 


\section{B. Solution Algorithm}

By using a Lagrange multiplier $\lambda \geq 0$, (3) can be converted into an unconstrained problem as,

$$
\begin{aligned}
& \min _{\{\boldsymbol{\mu}, \boldsymbol{\nu}, \boldsymbol{\sigma}\}} \sum_{i=1}^{A} E\left[D_{k}^{(n-i)}\left(\boldsymbol{\sigma}^{(n-i)}\right)\right]+\sum_{k=1}^{M} E\left[D_{k}^{(n)}(\boldsymbol{\mu}, \boldsymbol{\nu})\right] \\
&+\lambda\left\{\sum_{i=1}^{A} \sum_{k=1}^{M} \sigma_{k}^{(n-i)} T_{k}^{(n-i)}+\sum_{k=1}^{M} T_{k}^{(n)}\right\}
\end{aligned}
$$

The convex hull solution of this relaxed problem can be found by choosing an appropriate $\lambda$ to satisfy the transmission delay constraint. Techniques such as a bisection search or a fast convex search algorithm can be used to search for the appropriate $\lambda$ [13].

Given a specific $\lambda$, the minimization can be solved in three steps: bit allocation for retransmission, bit allocation for FEC and optimal mode selection for the current frame based the delay budget left. The first and second steps can be solved by using exhaustive search, and the optimal mode selection can be done by solving the following dynamic programming (DP) problem:

$\min _{\{\boldsymbol{\sigma} \in \mathcal{P}\}} \sum_{i=1}^{A} J^{(n-i)}\left(\boldsymbol{\sigma}^{(n-i)}\right)+\min _{\{\boldsymbol{\nu} \in \mathcal{R}\}}\left\{\min _{\{\boldsymbol{\mu} \in \mathcal{Q}\}} \sum_{k=1}^{M} J_{k}^{(n)}(\boldsymbol{\mu}, \boldsymbol{\nu})\right\}$, where $J^{(n-i)}=E\left[D_{k}^{(n-i)}\right]+\lambda \sum_{k=1}^{M} \sigma_{k}^{(n-i)} T_{k}^{(n-i)}$ and $J_{k}^{(n)}=E\left[D_{k}^{(n)}(\boldsymbol{\mu}, \boldsymbol{\nu})\right]+\lambda T_{k}^{(n)}$. The DP can be viewed as a shortest path problem in a trellis, where each stage corresponds to the mode selection for a given packet [14]. If the error concealment strategy does not introduce dependency across source packets, the Lagrangian in (4) is then separable. In this case, the time complexity would be $O\left(\left|2^{L} \times M \times\right| \mathcal{R} \times \mathcal{Q} \mid\right)$ [13], where $|\cdot|$ denotes the cardinality of the set inside, and $L$ is the number of lost packets in the optimization window.

\section{EXPERIMENTAL RESUlTS}

In the simulations, we choose an H.263+ codec [15] to perform source coding, and consider the QCIF $(176 \times 144)$ Foreman sequence. For error concealment, the lost $\mathrm{MB}$ is recovered from the MB with the same spatial location in the previously reconstructed frame. Rate control is not implemented in the work. Thus, every frame has the same transmission delay constraint, i.e., $T_{0}^{(n)}=T_{F}$. In all experiments, we set $A=4$, and $F=15$ fps.

Four schemes are compared: i) neither FEC nor retransmission (NFNR), ii) pure retransmission, iii) pure FEC, and iv) Hybrid FEC and selective Retransmission (HFSR). All four systems are optimized using the IJSCC framework.

\section{A. Sensitivity to RTT}

Figure 2 shows the performance of the four systems in terms of PSNR versus RTT with different level of channel loss rate. As shown, the HFSR system offers the best overall performance of the four. Retransmission is much more sensitive to RTT than FEC, as the performance of the pure retransmission scheme decreases dramatically as the network RTT gets longer.
In addition, we can see that retransmission is suitable for those applications where network RTT is short and channel loss rate is low, which confirms the observation in [3]. The disadvantage of retransmission when RTT gets longer comes from two sources: 1) Given the same value of $A$, which is decided by the initial setup time $T_{\max }$, the number of retransmission opportunities becomes less; 2) Errors accumulated due to error propagation from the motion compensation get larger, and consequently retransmission of lost packets becomes less efficient.

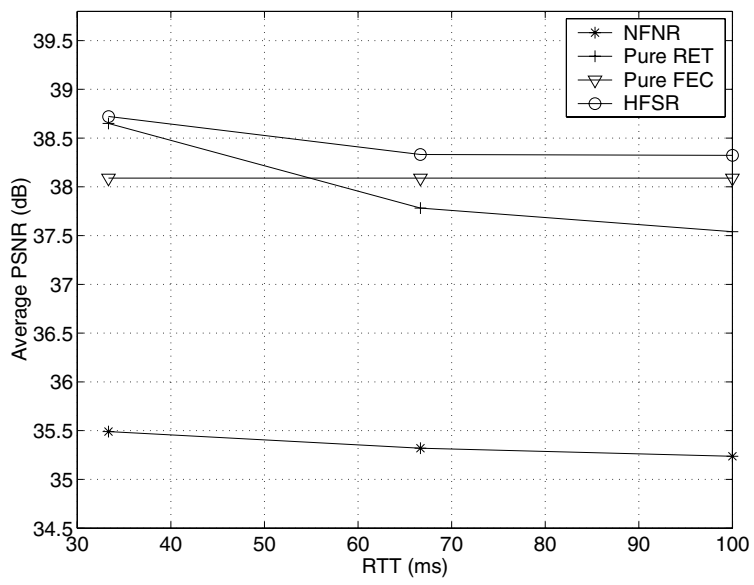

(a)

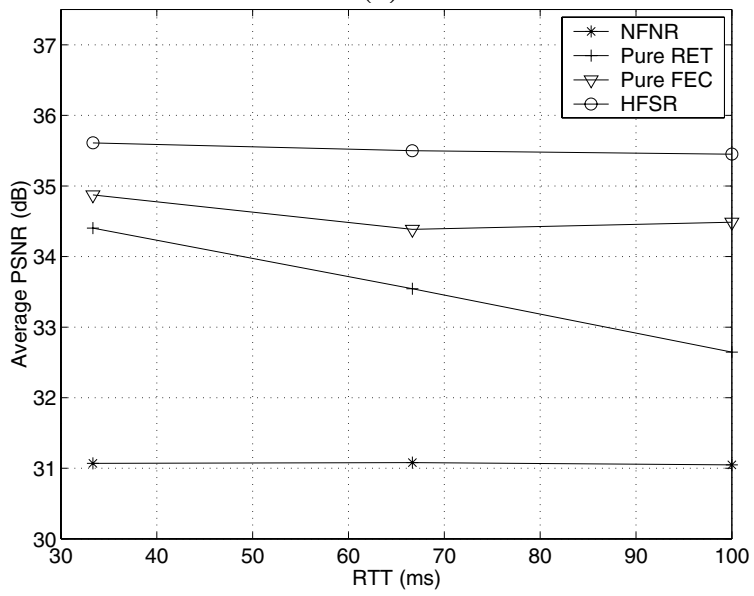

(b)

Fig. 2. Average PSNR vs. RTT, $R_{T}=480 \mathrm{kbps}, F=15$ fps (a) $\epsilon=0.02$ (b) $\epsilon=0.2$

\section{B. Sensitivity to packet loss rate}

In Fig. 3, we plot the performance of the four systems in terms of PSNR versus probability of transport packet loss when $R_{T}=480 \mathrm{kbps}, F=15 \mathrm{fps}$, and $\mathrm{RTT}=T_{F}$. It can be seen that the HFSR system achieves the best overall performance of the four. The resulting PSNR in the pure retransmission system drops faster than the pure FEC system, which means retransmission is more sensitive to packet loss rate. When the channel loss rate is high, FEC is more efficient since retransmission techniques need persistent retransmission to recover from packet loss, which results in large overhead. However, when the channel loss rate is small, retransmission 
becomes more efficient, since FEC typically requires a fixed amount of bandwidth overhead. Consequently, the pure retransmission system performs closely to the HFSR system at low $\epsilon$, as shown in Fig. 3.

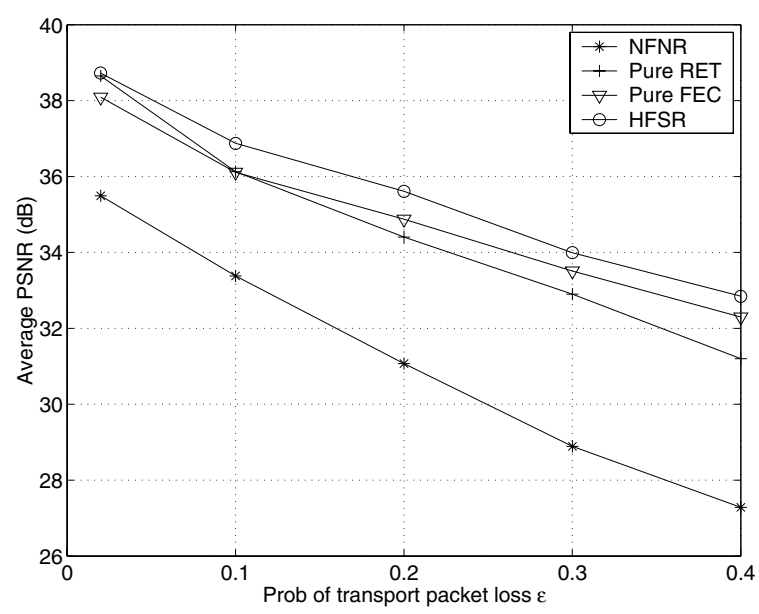

Fig. 3. Average PSNR vs. probability of transport packet loss $\epsilon, R_{T}=480$ kbps, $F=15$ fps, RTT $=T_{F}$.

\section{Sensitivity to transmission rate}

Figure 4 shows the performance of the four systems in terms of PSNR versus channel transmission rate when $\epsilon=0.2$, $F=15 \mathrm{fps}$ and $\mathrm{RTT}=T_{F}$. We can see that as the transmission rate increases, the PSNR of the pure FEC system increases faster than that of the pure retransmission system, which means that FEC is more sensitive to variations in the transmission rate. These observations imply that FEC is more efficient than retransmission when the transmission rate becomes greater (resulting in a higher bit budget per frame). This makes sense because FEC usually incurs constant overhead, which limits the use of FEC when the transmission rate is low.

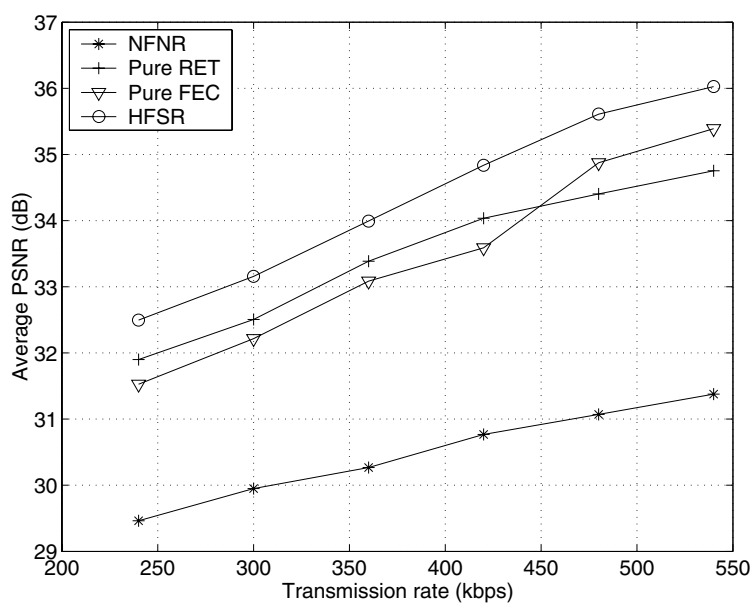

Fig. 4. Average PSNR vs. transmission rate $R_{T}, \epsilon=0.2, F=15$ fps, $\mathrm{RTT}=T_{F}$.

Although we only showed simulation results based on the QCIF Foreman sequence, extensive experiments have been carried out and similar results were obtained using other test sequences such as Akiyo, Container, and Carphone.

In summary, retransmission is suitable for short network RTT, low probability of packet loss, and low transmission rate, while FEC is more suitable otherwise. In general, our proposed hybrid FEC and selective retransmission scheme is able to find the best combination of the two.

\section{CONCLUSIONS}

In this paper, we studied the performance of different error correction schemes, such as FEC, ARQ, and hybrid FEC/selective retransmission. This study was carried out in the proposed IJSCC framework, which jointly considers the application layer error control components: error resilient source coding at the encoder, FEC and retransmission at the application layer, and error concealment at the receiver. Simulation results show that either FEC or retransmission may be more applicable in different situations. Improved results were obtained when the two were jointly employed in the proposed hybrid technique.

\section{REFERENCES}

[1] R. O. Hinds, T. N. Pappas, and J. S. Lim, "Joint block-based video source-channel coding for packet-switched networks," Proc. SPIE, vol. 3309, pp. 124-133, Jan. 1998.

[2] R. Zhang, S. L. Regunathan, and K. Rose, "Video coding with optimal inter/intra-mode switching for packet loss resilience," IEEE J. Select. Areas Commun., vol. 18, pp. 966-976, June 2000.

[3] F. Hartanto and H. R. Sirisena, "Hybrid error control mechanism for video transmission in the wireless IP networks," in Proc. of IEEE Tenth Workshop on Local and Metropolitan Area Networks (LANMAN'99), Sydney, Australia, Nov. 1999.

[4] P. A. Chou, A. E. Mohr, A. Wang, and S. Mehrotra, "Error control for receiver-driven layered multicast of audio and video," IEEE Trans. Multimedia, pp. 108-122, March 2001.

[5] D. Wu, Y. T. Hou, and Y-Q Zhang, "Transporting real-time video over the Internet: Challenges and approaches," Proc. IEEE, vol. 88, pp. 18551877, Dec. 2000.

[6] Y. Wang, G. Wen, S. Wenger, and A. K. Katsaggelos, "Review of error resilience techniques for video communications," IEEE Signal Processing Magazine, vol. 17, pp. 61-82, July 2000.

[7] M. Gallant and F. Kossentini, "Rate-distortion optimized layered coding with unequal error protection for robust Internet video," IEEE Trans. on Circ. and Syst. for Video Techn., vol. 11, pp. 357-372, March 2001.

[8] F. Zhai, Y. Eisenberg, T. N. Pappas, R. Berry, and A. K. Katsaggelos, "Packetization schemes for forward error correction in Internet video streaming," in Proc. 41st Allerton Conf. on Communiciation, Control and Computing, Otc. 2003.

[9] P. A. Chou and Z. Miao, "Rate-distortion optimized streaming of packetized media," IEEE Trans. on Multimedia, 2001, Submitted.

[10] S. Falahati, A. Svensson, N. C. Ericsson, and A. Ahlen, "Hybrid type-II ARQ/AMS and scheduling using channel prediction for downlink packet transmission on fading channels," in Nordic Radio Symposium, 2001.

[11] G. J. Wang, Q. Zhang, W. W. Zhu, and Y.-Q. Zhang, "Channel-adaptive error control for scalable video over wireless channel," in IEEE MoMuc 2000 , Oct. 2000.

[12] M. Podolsky, M. Vetterli, and S. McCanne, "Limited retransmission of real-time layered multimedia," in Proc. IEEE Workshop Multimedia Signal Processing, Dec. 1998, pp. 591-596.

[13] G. M. Schuster and A. K. Katsaggelos, Rate-Distortion Based Video Compression: Optimal Video Frame Compression and Object Boundary Encoding, Kluwer Academic Publishers, 1997.

[14] F. Zhai, C. E. Luna, Y. Eisenberg, T. N. Pappas, R. Berry, and A. K. Katsaggelos, "Joint source coding and packet classification for real-time video transmission over differentiated services networks," IEEE Trans. Multimedia, 2004, accepted.

[15] ITU-T, Video coding for low bitrate communication, ITU-T Recommendation H.263, Jan. 1998, Version 2. 